Somnologie $2016 \cdot 20: 322$

DOI 10.1007/s11818-016-0091-3

Online publiziert: 26. Oktober 2016

(c) Springer-Verlag Berlin Heidelberg 2016

\title{
Die Zeitschrift Somnologie dankt den Gutachtern 2016
}

Die Schriftleitung und die Redaktion danken den Herausgebern der Zeitschrift und insbesondere allen weiteren Experten und Expertinnen für ihre Unterstützung im Jahr 2016 als wissenschaftliche Gutachter für die Zeitschrift Somnologie. Durch ihren Einsatz garantieren sie im Rahmen des Peer-Review-Verfahrens der eingereichten Manuskripte die hohe Qualität der Zeitschrift.

Philipp Arens, Berlin

Sven Armbrust, Neubrandenburg

Thomas Bitter, Oeynhausen

Alexander Blau, Berlin

Stefan Cohrs, Rostock

Nancy Digdon, Edmonton, Canada

Alexander Dück, Rostock

Stephan Eichholz, Dresden

Gisela Erdmann, Berlin

Thomas Erler, Cottbus

Ingo Fietze, Berlin

Henrik Fox, Bad Oeynhausen

Wolfgang Galetke, Köln

Peter Geisler, Regensburg

Martin Glos, Berlin

Refika Hamutcu Istanbul, Turkey

Holger Hein, Reinbek

Matthias Hölzl, Traunstein

Helmut Hoesl, Regensburg

Tina In-Albon, Koblenz, Landau

Frank Kirchhoff, Rostock

Karsten Klementz, Potsdam

Sylvia Kotterba, Lehr

Naima Laharnar, Berlin

Helena Larramona, Sabadell, Spain

Joachim T. Maurer, Mannheim
Geert Mayer, Schwalmstadt-Treysa

Silvia Miano, Lugano, Schweiz

Andreas Müller, Sebnitz

Silvia Müller-Hagedorn, Tübingen

Georg Nilius, Hagen-Ambrock

Winfried Randerath, Solingen

Christoph Randler, Tübingen

Farid Salih, Berlin

Sebastian Scharfe, Kassel

Angelika Schlarb, Bielefeld

Hartmut Schulz, Erfurt

Justus Schwabedal, Dresden

Lisa Schwarz, Berlin

Ulrich Sommer, Mannheim

Kai Spiegelhalder, Freiburg

Frank Stemberg, Worms

Boris A. Stuck, Essen

Michael S. Urschitz, Mainz

Christian Veauthier, Berlin

Silke Weber, São Paulo, Brazil

Hans-Günter Weeß, Klingenmünster

Gerhard Weinreich, Essen

Peter Young, Münster

Josef Zeitlhofer, Wien

Sandra Zimmermann, Berlin 